\title{
Mathematical and Computational and Modeling for Leprosy's Dynamics
}

\section{Modelagem matemática e computacional para a dinâmica da hanseníase}

Claudia Mazza Dias ${ }^{1}$, Dayse Haime Pastore², Erito Marques de Souza Filho³

\section{RESUMO}

Este artigo trata da modelagem matemática da hanseníase, doença infecciosa e contaminante considerada negligenciada. Um modelo compartimental é analisado na investigação da dispersão da hanseníase em uma população, a fim de prever sua transmissão a novos pacientes e permitindo que diferentes grupos de indivíduos sejam analisados em face à exposição à infecção, buscando o impacto no entendimento da transmissão e planejamento de estratégias de controle da doença.

Palavras-chave: Biomatemática, Modelagem Computacional em Saúde, Modelagem Matemática e Aplicações

\section{ABSTRACT}

This paper deals with the mathematical modeling of leprosy, an infectious and contagious disease considered neglected. A compartmental model is analyzed in the investigation of the dispersion of leprosy in a population in order to predict the transmission of new patients and allowing different groups of individuals to be analyzed in the face of exposure and infection, seeking the impact on the understanding of transmission and planning of disease control strategies.

Keywords: Biomathematics, Computational Modeling in Health, Mathematical Modeling and Applications
Doutora em Engenharia Civil. Professora da Universidade Federal Rural do Rio de Janeiro (UFRRJ).

E-mail: mazzaclaudia@gmail.com

${ }^{2}$ Doutora em Ciências. Professora do Centro Federal de Educação Tecnológica (CEFET-RJ).

3 Doutor em Engenharia de Produção. Professor da Universidade Federal Rural do Rio de Janeiro (UFRRJ). 


\section{INTRODUCTION}

According to AZULAY (2015) "leprosy is an infectious-contagious disease caused by Mycobacterium leprae affecting mainly skin and or peripheral nerves. The disease can affect virtually all organs and systems in which there are macrophages, except the central nervous system. The disease progresses in a chronic way, and may present acute episodes called reactions. It is potentially disabling and, although curable, its diagnosis causes great psychosocial impact, due to the prejudices and stigmas that have been involved since antiquity". Although it has a high success rate in treatment in the vast majority of cases, countries with a high incidence of cases are generally the least developed or with poor hygiene conditions and overpopulation. Just in 2011, the Ministry of Health registered more than 33,000 cases of the disease in Brazil (BRAZILIAN SOCIETY OF DERMATOLOGY, 2015). The use of mathematical models is recognized as useful in the prevention, understanding of the dynamics and incidence of diseases, as well as in the planning of control actions (MURRAY, 2002). This work analyzes a compartmental model in the investigation of leprosy dispersal in a population aiming to predict the transmission to new patients and to allow the analysis of different groups of individuals in the face of exposure to infection. For this, it uses a conceptual model (LECHAT et al.,1985; MEIMA et al., 1999; BLOCK et al., 2015) to describe the dynamics of the disease. Thus, a mathematical model is constructed based on the conceptual model, and the resulting system of ordinary differential equations is solved numerically.

\section{MODELING}

Taking as a starting point the conceptual schematic model proposed by MEIMA et al. (1999), which considers the heterogeneity in susceptibility and the incubation period, constituting an advance at the time, this very complex model was presented only in schematic form as can be seen at Figure 1. BLOCK et al. (2015) presented a survey on the models developed so far. One aspect that is evident is that the papers do not point out clearly the solution strategies. In the words of the same authors "mathematical models in leprosy have not been applied extensively. This is in part due to the limited size of the leprosy problem in terms of numbers and health burden compared to many other infectious diseases such as HIV/AIDS, tuberculosis and malaria. Even within the group of neglected tropical diseases, the contribution of leprosy is modest. Few scientists have taken lasting interest in 
leprosy and funding for research is limited." Despite the apparent lack of attention with leprosy, cases have multiplied in our country. In a recent study, researchers from the Mamirauá reservoir in Amazônia state that: “... since the region is endemic with a high risk of malaria transmission, tuberculosis and leprosy (six cases). The incidence of leprosy is high in Uarini (15.5 / 100,000 inhabitants in 2015) and hyperendemic in Fonte Boa (47 / 100,000 inhabitants in 2015)" (MEDEIROS et al., 2018). The present work contributes to the research on the subject, proposing that, based on the schematic model already mentioned, the corresponding system of equations, presented below, can be solved, for example, by numerical methods (BURDEN \& FAIRES, 2011).

\subsection{THE MATHEMATICAL MODEL}

The corresponding mathematical model can be described as,

$$
\begin{gathered}
\frac{d A}{d t}=f_{c a}-f_{z} A \\
\frac{d B}{d t}=f_{c b}-f_{b d} B D+f_{d b} D+f_{e b} E+f_{i b} I-f_{z} B \\
\frac{d C}{d t}=f_{a c} C-f_{c d} C D-f_{z} C \\
\frac{d D}{d t}=-f_{d b} D-f_{c d} C D+F_{b d} B D-f_{d e} D-f_{d h} D-f_{d f} D-f_{d g} D-f_{z} D \\
\frac{d E}{d t}=f_{d e} D-f_{e b} E-f_{e h} E-f_{e i} E+F_{j e} J-f_{z} E \\
\frac{d F}{d t}=f_{d f} D+f_{i f} J-f_{j i} F-f_{j g} F-f_{z} F \\
\frac{d G}{d t}=f_{f g} F+f_{d g} D+f_{j g} J-f_{g i} G-f_{z} G \\
\frac{d H}{d t}=f_{e h} E+f_{d h} D-f_{z} H \\
\frac{d I}{d t}=f_{e i} E+f_{f i} F+f_{g i} G-f_{i b} I-f_{i j} I-f_{z} I \\
\frac{d J}{d t}=-f_{j e} J-f_{i f} J-f_{j g} J+f_{i j} I-f_{z} J
\end{gathered}
$$

In the equations (1), $t$ represents the analysis time, the capital letters correspond to the compartments or populations. Thus, $A$ represents population with natural immunity, B, 


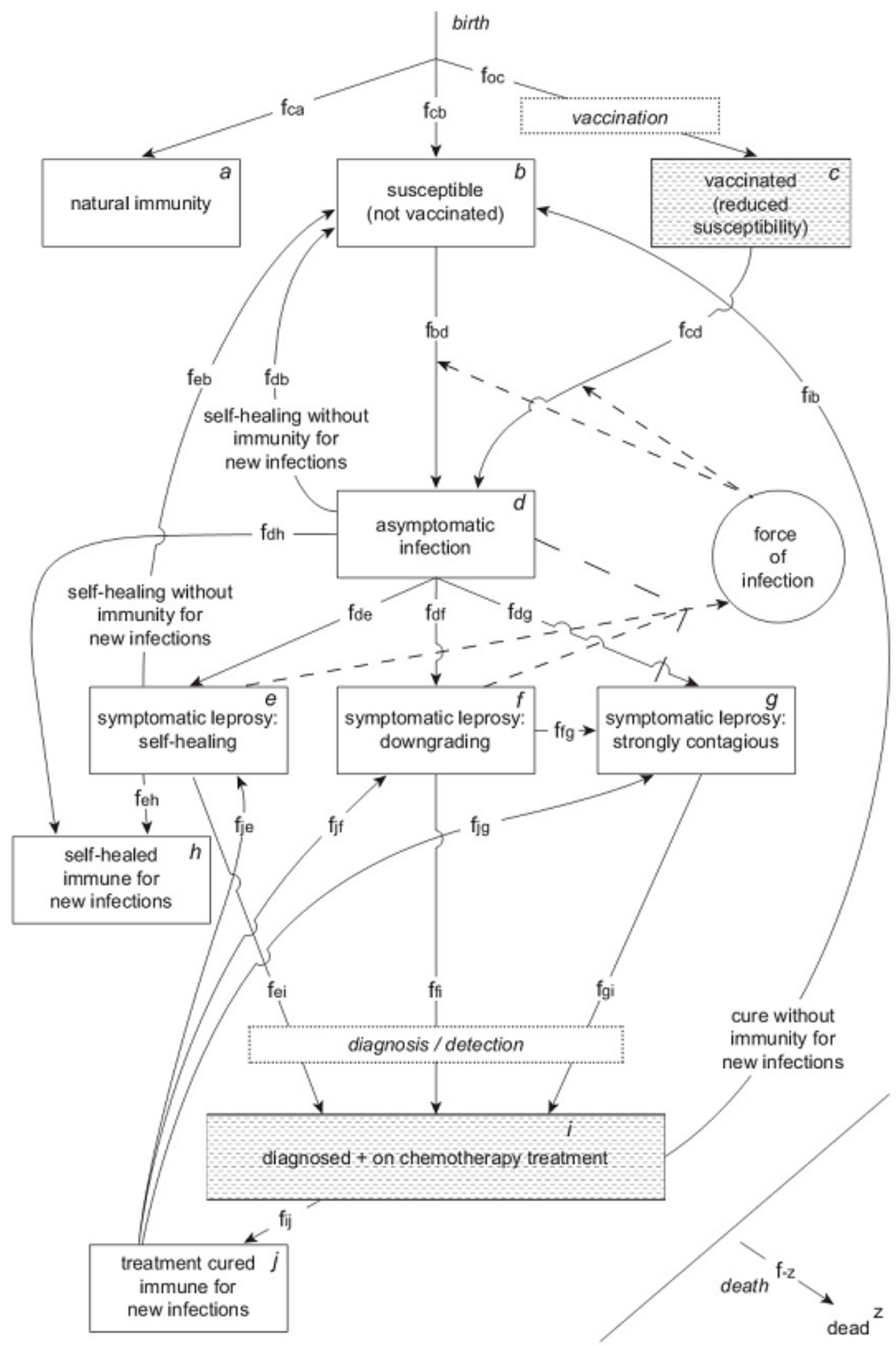

Figure 1. Scheme of Leprosy Dynamics by MEIMA et al. (1999) Apud: BLOCK et al. (2015).

susceptible population (not vaccinated); $C$, vaccinated population (BCG) with reduced susceptibility. It is well known that the vaccine provides partial protection against leprosy. $D$ 
is the population with asymptomatic infection; $E$, population with symptomatic leprosy: selfhealing; $F$, population with symptomatic leprosy: downgrading; $G$, population with symptomatic leprosy: strongly contagious; $H$, population self-healed immune for new infections; I, population diagnosed and treated by polychemotherapeutic; e finally, $J$ corresponds to population cured and immune for new infections.

Lowercase letters correspond to transfer rates between compartments, so: $f_{c a}$ represents the natural immunity tax; $f_{c b}$, the rate of population that was not vaccinated; $f_{a c}$ , vaccination rate; $f_{e b}$, the corresponding tax of population not immune and consequently susceptible; $f_{b d}$, asymptomatic population that turn self-healing without immunity rate; $f_{d b}$ , susceptible population that turn asymptomatic infected rate; $f_{c d}$, vaccinated population that turn asymptomatic infected rate; $f_{\text {is }}$, population cured after diagnostic and treatment rate; $f_{d h}$, asymptomatic patients that turn self-healing immune for new infections rate; $f_{d e}$ , asymptomatic patients that turn symptomatic self-healing rate; $f_{a^{\prime}}$, asymptomatic patients that turn symptomatic downgrading rate; $f_{d g}$, asymptomatic patients that turn symptomatic strongly contagious rate; $f_{\text {sis }}$, downgrading patient that turn strongly contagious rate; $f_{\text {eh }}$, symptomatic patients self-healed immune for new infections rate; $f_{e i}$, symptomatic patients self-healed diagnosed and treated rate; $f_{f_{i}}$, symptomatic patients downgraded diagnosed and treated rate; $f_{g i}$, symptomatic patients strongly contagious diagnosed and treated rate; $f_{j e}$, cured by treatment patients turn symptomatic self-healed rate; $f_{j f}$, cured by treatment patients turn symptomatic downgraded rate; $f_{j g}$, cured by treatment patients turn symptomatic strongly contagious rate; $f_{i j}$, diagnosed and cured patients cured and immune for new infections rate. $f_{z}$ is the mortality tax.

Although the dynamic system (1) closely matches the scheme of Figure 1, it is clear that separation of the population into different groups at birth does not faithfully represent reality, where the vaccine is given at birth, but can be given up to five years of age, when the cost $\mathrm{x}$ benefit ratio is maintained, as recommended by the Brazilian Ministry of Health. Thus, the model proposed here eliminates the $A$ compartment, corresponding to individuals with natural immunity, understanding that the whole population is born immune 
and part of it is vaccinated and part is not vaccinated (MANUAL DE NORMAS DE VACINAÇÃO, 2001). Thus, the dynamic system follows:

$$
\begin{gathered}
\frac{d B}{d t}=f_{c b}-f_{b c} B-f_{b d} B D+f_{d b} D+f_{e b} E+f_{i b} I-f_{z} B \\
\frac{d C}{d t}=f_{b c} B-f_{c d} C D-f_{z} C \\
\frac{d D}{d t}=-f_{d b} D-f_{c d} C D+F_{b d} B D-f_{d e} D-f_{d h} D-f_{d f} D-f_{d g} D-f_{z} D \\
\frac{d E}{d t}=f_{d e} D-f_{e b} E-f_{e h} E-f_{e i} E+F_{j e} J-f_{z} E \\
\frac{d F}{d t}=f_{d f} D+f_{j f} J-f_{f i} F-f_{f g} F-f_{z} F \\
\frac{d G}{d t}=f_{f g} F+f_{d g} D+f_{j g} J-f_{g i} G-f_{z} G \\
\frac{d H}{d t}=f_{e h} E+f_{d h} D-f_{z} H \\
\frac{d I}{d t}=f_{e i} E+f_{j i} F+f_{g i} G-f_{i b} I-f_{i j} I-f_{z} I \\
\frac{d J}{d t}=-f_{j e} J-f_{i f} J-f_{j g} J+f_{i j} I-f_{z} J \\
\text { Where } f_{b c} \text { represents vaccination tax. }
\end{gathered}
$$

\section{NUMERICAL EXPERIMENTS}

For the numerical experiments, Table 1 shows the input parameters. Part of the parameters were adapted from the literature (MEIMA et al., 1999) and part used IBGE (2018) data for an estimate of birth and death rates in Brazil.

Figures 2 to 10 show the results over the analysis time (70 years) for each compartment, or population considered in the model (2). It was considered a population of 1000 inhabitants, exposed to the presence of a single sick individual. From the analysis of the results it is possible to see that the system reproduces the expected dynamics for the disease through a scenario where the majority of the population (about 90\%) have access to $B C G$ vaccination, but vaccination does not guarantee $100 \%$ immunity the disease. Thus, many of those who have contact with the disease do not get sick and among those who remain asymptomatic, many are cured without treatment. This situation, assumed by the modeling, is very frequent. The next step of the analysis will require more realistic parameters, based on time series of data. The following initial values were considered for the populations: $B(0)=500 ; C(0)=499 ; D(0)=1 ; E(0)=0 ; F(0)=0 ; G(0)=0 ; H(0)=0 ; 1$ (0) $=0$; and $J(0)=0$ 
Table 1. Input Parameters.

\begin{tabular}{cccccc}
\hline Parameter & Value & Parameter & Value & Parameter & Value \\
\hline$f_{c b}$ & 0.0014 & $f_{f i}$ & 0.08 & $f_{d h}$ & 0.03 \\
$f_{e b}$ & 0.033 & $f_{j e}$ & 0.01 & $f_{d f}$ & 0.021 \\
$f_{b d}$ & 0.00075 & $f_{j g}$ & 0.04 & $f_{f g}$ & 0.02 \\
& & & & & \\
$f_{i b}$ & 0.05 & $f_{z i}$ & 0.0006 & & 0.067 \\
$f_{d e}$ & 0.042 & $f_{b c}$ & 0.09 & $f_{g i}$ & 0.1 \\
$f_{d g}$ & 0.007 & $f_{d b}$ & 0.03 & $f_{i f}$ & 0.05 \\
$f_{e h}$ & $0 ; 033$ & $f_{c d}$ & $0.5 f_{c b}$ & $f_{i j}$ & 0.05 \\
\hline
\end{tabular}

\section{CONCLUSIONS}

The paper presents a mathematical model for the dynamics of leprosy and investigates the applicability of the conceptual model of MEIMA et al. (1999) modified to better representation. From the observation of the numerical results, the model can be an important tool to control the transmission of leprosy, a disease considered neglected, that is, endemic in low-income populations and with reduced investments in research, production of drugs and in its control. The model is capable of investigating certain uncertainties in the epidemiology of the disease and considers natural immunity, incubation period, asymptotic infection, among other factors, although new numerical experiments are needed from more realistic data for the estimation of the parameters used. Its mathematical development and numerical treatment are important steps in the development of new models that mainly consider heterogeneity in susceptibility and new advances in the mechanisms of natural immunity and the susceptibility of paucibacillary and multibacillary forms. Although it is a compulsory notification disease in Brazil, it still requires a better data collection, since the current data are contradictory and deserve better quality records.

The main aim of the research is to develop mathematical models that describes the dynamics of the disease well, seeking an impact important in the understanding of its 
transmission and in the planning of a strategy of control. Mathematical models allow a discussion of the most important factors in this process and the design of strategies for the use of medicines that minimize the resistance to medication, for example.

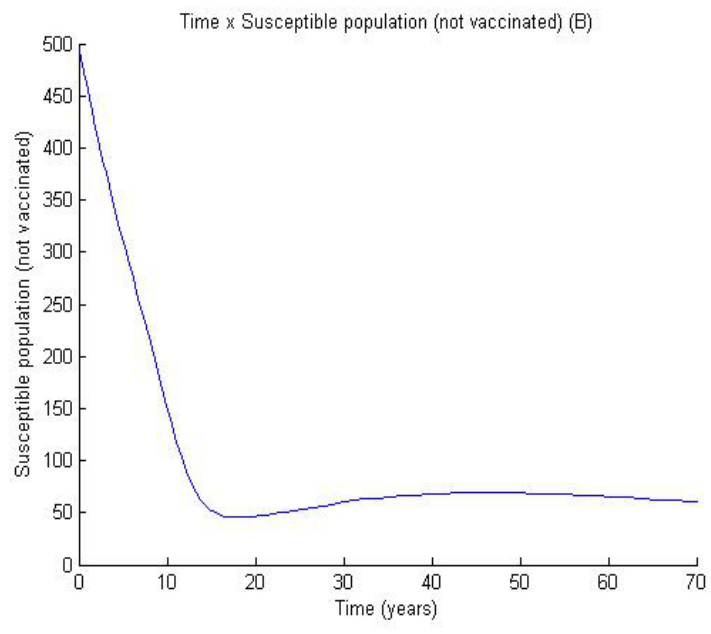

Figure 2. Susceptible population (not vaccinated) - $B$.

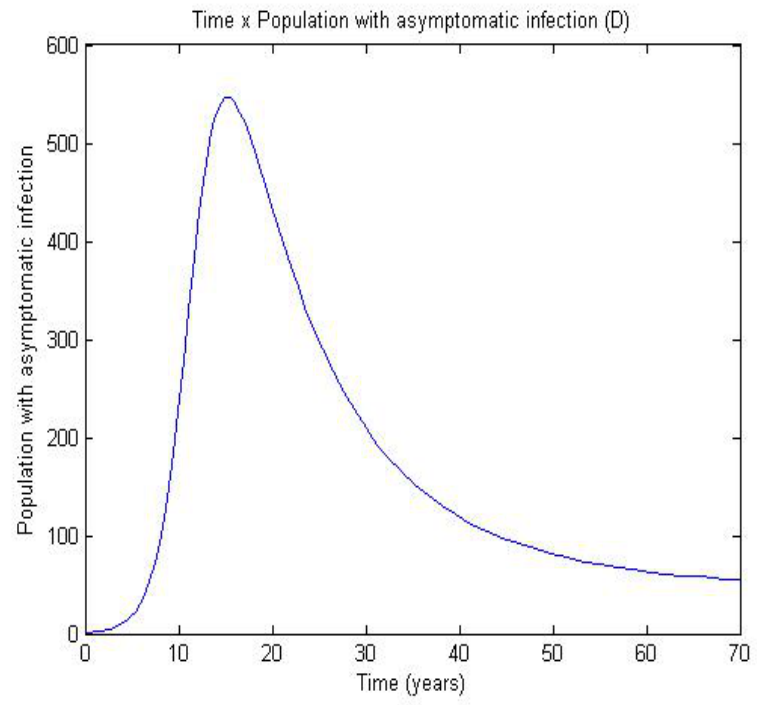

Figure 4. Population with asymptomatic infection $-D$.

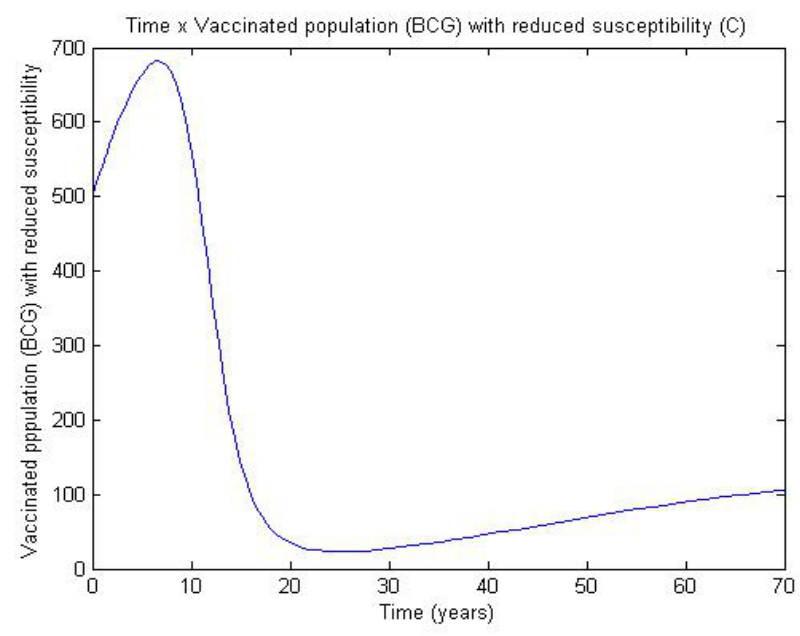

Figure 3. Vaccinated population (BCG) with reduced susceptibility $-C$.

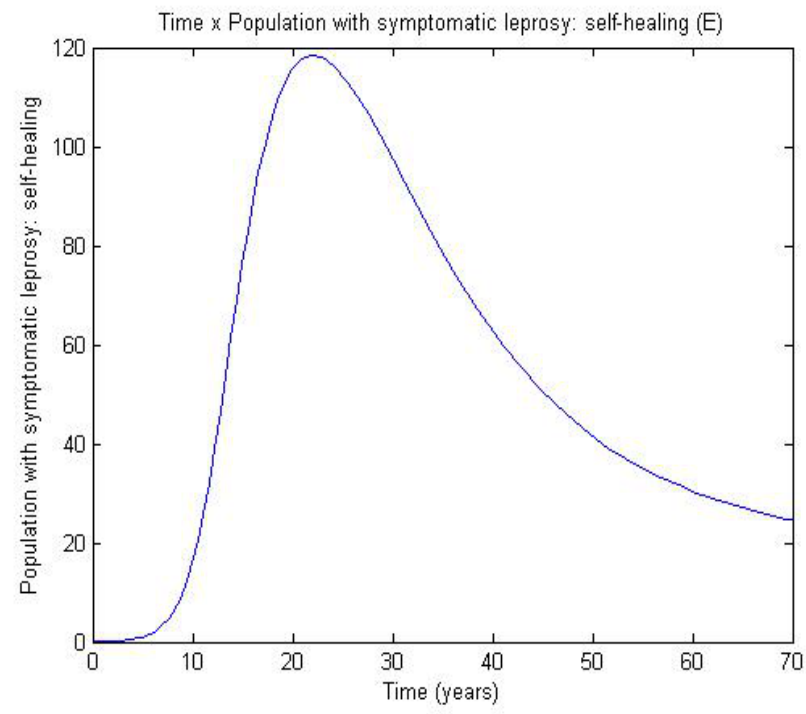

Figure 5. Population with symptomatic leprosy: self-healing $-E$. 


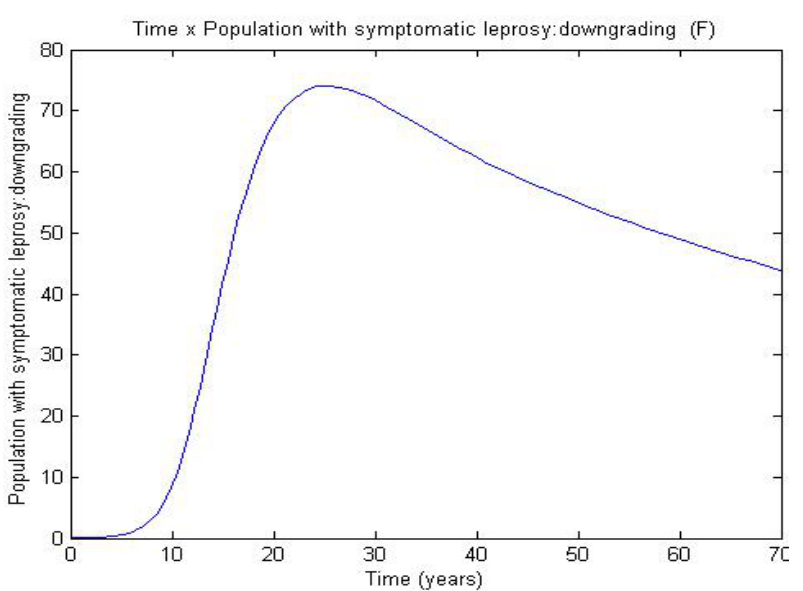

Figure 6. Population with symptomatic leprosy: downgrading $-F$.

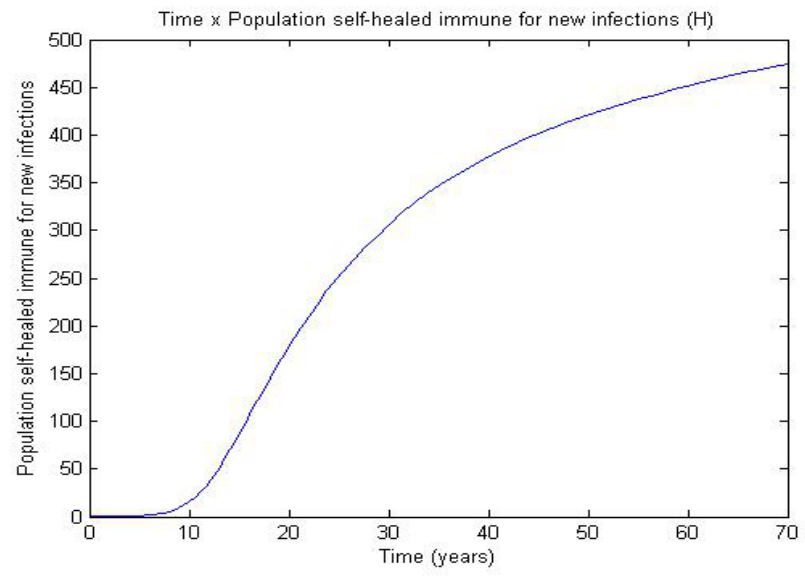

Figure 8. Population self-healed immune for new infections- $H$.

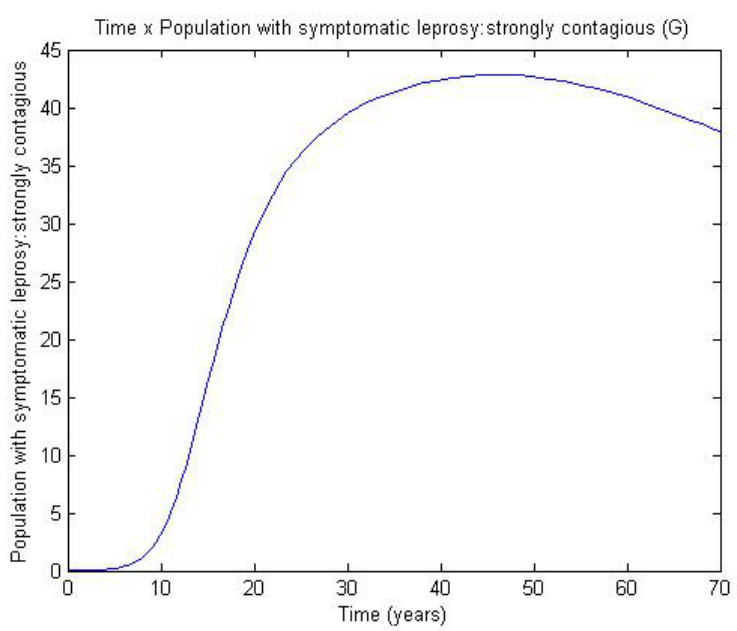

Figure 7. Population with symptomatic leprosy: strongly contagious- $G$.

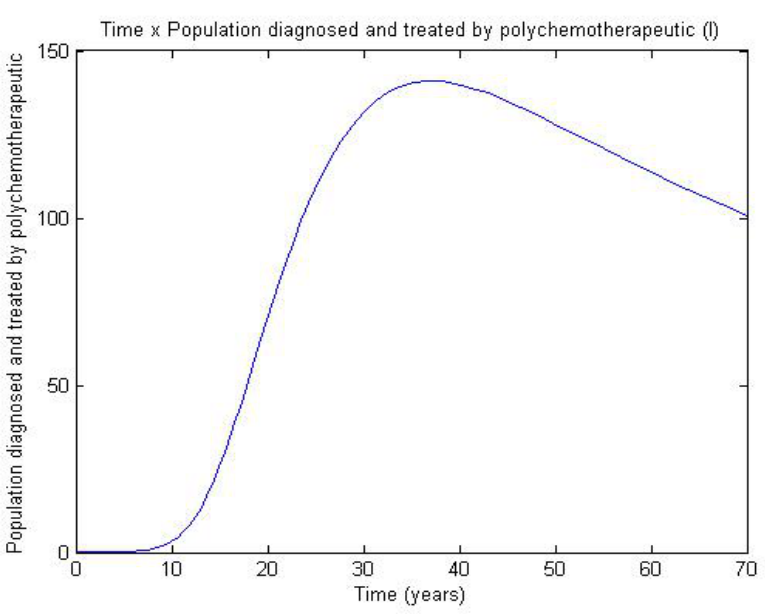

Figure 9. Population diagnosed and treated by polychemotherapeutic- $I$.

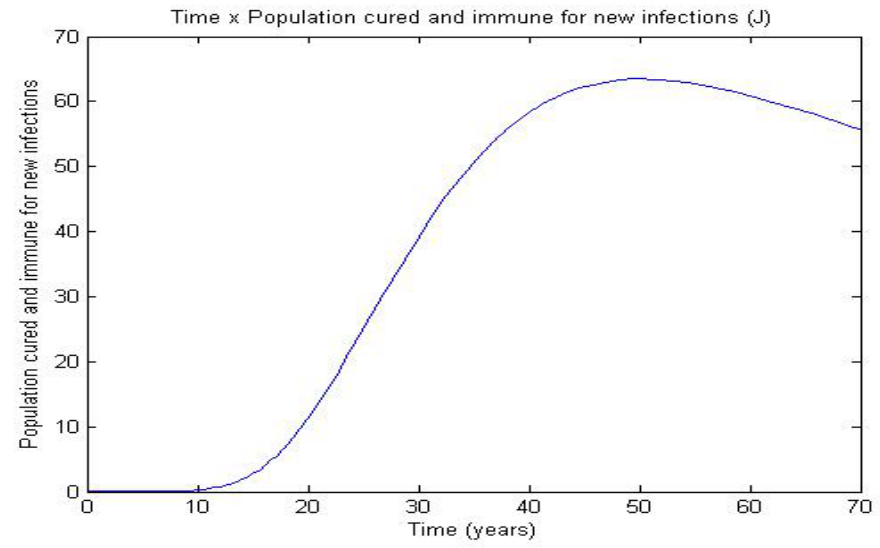

Figure 10. Population cured and immune for new infections- $J$. 


\section{Acknowledgements}

This study was financed in part by the Coordenação de Aperfeiçoamento de Pessoal de Nível Superior - Brasil (CAPES).

\section{REFERENCES}

AZULAY, R. D.Dermatologia, 6a ed.,Guanabara Koogan, Rio de Janeiro, 2015.

BLOCK, D. J.; FISCHER, E. A. J.; RICHARDUS, J. H., Mathematical Modelling of Leprosy and Its Control. Advances in Parasitology, 33-51, 2015.

\section{BRAZILIAN SOCIETY OF DERMATOLOGY. Website:} <http://www.sbd.org.br/doencas/hanseniase>. Accessed in: Jun 06, 2015.

BRAZILIAN INSTITUTE OF GEOGRAPHY AND STATISTICS. Website: <https://www.ibge.gov.br/>. Accessed in: July 10, 2018.

BURDEN, R. L.; FAIRES, J. D., Numerical Analysis, 9th ed., Cengage Learning, 2011.

LECHAT, M. F.; MISSON, C. B.; LAMBERT, A., Simulation of vaccination and resistance in leprosy using an epidemiometric model. International Journal of Leprosy and Other Mycobacterial Disease, 53, 461-467, 1985.

MANUAL DE NORMAS DE VACINAÇÃO. Ministério da Saúde. Brasília, 2001. Website: <http://bvsms.saude.gov.br/bvs/publicacoes/funasa/manu_normas_vac.pdf> Accessed in: Jan 21, 2019.

MEDEIROS, M. S.; AUGUSTO, L. G. S.; BARCA, S.; SACRAMENTO, D. S.; NETA, I. S. S.; GONÇALVES, I. C.; COSTA, A. M., A saúde no contexto de uma reserva de desenvolvimento sustentável: o caso de Mamirauá, na Amazônia Brasileira. Saúde e Sociedade, 27, 1, 128-148, 2018.

MEIMA, A.; GUPTE, M. D.; VAN OORTMARSEEN, G. J.; HABBEMA, J. D. F., SIMLEP: a simulation model for leprosy transmission and control. International Journal of Leprosy and Other Mycobacterial Disease, 67, 215-236, 1999.

MURRAY, J. D., Mathematical Biology, $1^{\text {st }}$ ed., Springer-Verlang, Berlin, 2002. 